\title{
Upregulated microRNA-16 as an oncogene in renal cell carcinoma
}

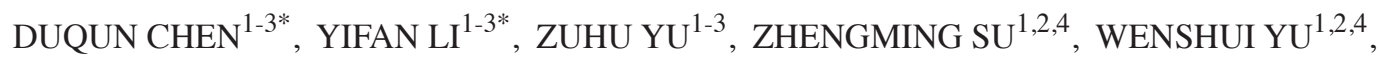 \\ YUCHI LI ${ }^{1,2,4}$, SHANGQI YANG ${ }^{1,2}$, YAOTING GUI ${ }^{2}$, LIANGCHAO NI ${ }^{1,2}$ and YONGQING LAI ${ }^{1,2}$ \\ ${ }^{1}$ Department of Urology, Peking University Shenzhen Hospital, Shenzhen, Guangdong 518036; ${ }^{2}$ Guangdong and Shenzhen \\ Key Laboratory of Male Reproductive Medicine and Genetics, Institute of Urology, Shenzhen PKU-HKUST Medical \\ Center, Shenzhen, Guangdong 518036; ${ }^{3}$ Department of Urology, Anhui Medical University, Hefei, Anhui 230032; \\ ${ }^{4}$ Department of Urology, Shantou University Medical College, Shantou, Guangdong 515041, P.R. China
}

Received May 7, 2014; Accepted February 11, 2015

DOI: $10.3892 / \mathrm{mmr} .2015 .3496$

\begin{abstract}
MicroRNAs (miRs) are small, endogenous noncoding RNAs that serve a significant function in various biologic processes, including those involved in cancer. The present study aimed to determine the expression and function of miR-16 in renal cell carcinoma (RCC). Quantitative polymerase chain reaction was used to quantify the expression of miR-16 in 48 paired RCC tissues and adjacent normal tissues. The impact of miR-16 on cell proliferation, migration and apoptosis was analyzed by transfecting miR-16 mature molecules into the renal cancer cell lines 786-O and ACHN. The results indicated that miR-16 was significantly upregulated in RCC tissues $(\mathrm{P}<0.05)$. Downregulation of miR-16 resulted in reduced cell proliferation and migration and increased levels of apoptosis, while overexpression of miR-16 resulted in accelerated cellular proliferation and migration, suggesting that miR-16 may function as an oncogene in RCC. The present study demonstrated for the first time, to the best of our knowledge, that miR-16 is upregulated in RCC and acts as an oncogene by inducing cellular proliferation, migration and reducing apoptosis. Further study of miR-16 in RCC may clarify the molecular mechanisms of RCC carcinogenesis and aid in the development of novel biomarkers and therapeutic options.
\end{abstract}

Correspondence to: Professor Yongqing Lai or Professor Liangchao Ni, Department of Urology, Peking University Shenzhen Hospital, 1120 Lianhua Road, Shenzhen, Guangdong 518036, P.R. China

E-mail: yqlord@163.com

E-mail: niliangchao@yahoo.cn

*Contributed equally

Key words: microRNA-16, oncogene, renal cell carcinoma

\section{Introduction}

Renal cell carcinoma (RCC) is the most common type of malignancy in kidney parenchyma, with a high rate of recurrence and mortality (1). Over the last two decades, the incidence of RCC has progressively increased and at present, it accounts for $3 \%$ of all cases of cancer (2). Early diagnosis of RCC is a challenge, as it presents no clinical symptoms for the majority of its course (3). Almost $30 \%$ of all patients exhibit metastatic disease at presentation, and $40 \%$ of patients with localized disease ultimately develop distant metastases following removal of the primary tumor (4). Therefore, it is critical to identify novel molecular mechanisms, including microRNAs (miRs), to elucidate RCC oncogenesis and metastasis.

miRs are non-coding RNAs of $\sim 22$ nucleotides in length, which function as regulators at the post-transcriptional level (5). Numerous studies have demonstrated the notable roles of miRs in cancer pathogenesis, including onset, progression and metastasis $(6,7)$. Involvement of miRs in the oncogenic process has been indicated by the observation that the 13 q14 deletion characterizing $>50 \%$ of all cases of chronic lymphocytic leukemia results in a loss of miR-16 genes (8). This finding provided the primary evidence that miR genes may have a role in tumorigenesis. Downregulation of miR-16 has been reported in other neoplastic malignancies, including multiple myeloma (9), pituitary adenomas (10), mantle cell lymphoma (11), lung cancer (12) and prostate carcinoma (13).

miR-16 is involved in numerous types of cancer; thus, the present study investigated the potential role and function of miR-16 in RCC. Quantitative polymerase chain reaction (qPCR) was used to quantify the expression of miR-16 in RCC tissues and paired normal adjacent tissues, and the impact of miR-16 on renal cancer was assessed by MTT, wound scratch and apoptosis assays following transfection of mature miR-16 molecules. Bioinformatics analysis was also performed to predict the target genes of miR-16.

\section{Materials and methods}

Human tissue samples and cell lines. All human RCC tissues and adjacent normal tissues were collected from Anhui Medical 
University (Hefei, China) and Peking University Shenzhen Hospital (Shenzhen, China), between January 2012 and August 2013. Written informed consent was obtained from each patient prior to sample collection. The collection and use of these samples were reviewed and approved by the ethics committees of the First Affiliated Hospital of Anhui Medical University (Hefei, China) and Peking University Shenzhen Hospital (Shenzhen, China). Once dissected, fresh tissues were immersed in RNAlater RNA stabilization reagent (Qiagen GmbH, Hilden, Germany) within $30 \mathrm{~min}$ then stored at $-80^{\circ} \mathrm{C}$. The pathological and clinical characteristics of 48 RCC patients are presented in Table I. The age range of patients was 29-76 years, with a median age of 52 years.

The two human renal cancer cell lines ACHN and 786-O were used in the present study. Cells were cultured at $37^{\circ} \mathrm{C}$ in Dulbecco's modified Eagle's medium (Gibco-BRL, Invitrogen Life Technologies, Carlsbad, CA, USA) mixed with 10\% fetal bovine serum (Invitrogen Life Technologies) in an incubator with a $5 \% \mathrm{CO}_{2}$ atmosphere.

RNA isolation and reverse transcription (RT)- $q P C R$. According to the manufacturer's instructions, total RNA from each sample was extracted using TRIzol reagent (Invitrogen Life Technologies, Carlsbad, CA, USA) and purified using an RNeasy Maxi kit (Qiagen $\mathrm{GmbH}$ ). The RT reaction was conducted with $1 \mu \mathrm{g}$ total RNA from each sample to obtain the cDNA templates, using a miScript RT kit (Qiagen $\mathrm{GmbH}$ ) according to the manufacturer's instructions. The qPCR reaction was performed in a LightCycler 480 Real-Time PCR system (Roche Diagnostics, Basel, Switzerland) using miScript SYBR Green PCR kit (Qiagen GmbH) with U6 small nuclear RNA (Qiagen $\mathrm{GmbH}$ ) as a control. The reaction mixture consisted of the following reagents: Specific microRNA primer $(1 \mu \mathrm{l})$; cDNA template $(1 \mu 1)$; 2X QuantiTect SYBR Green PCR Master Mix (10 $\mu$ l); 10X miScript Universal Primer ( $2 \mu 1)$; and RNase-free water to a total volume of $20 \mu \mathrm{l}$. The reverse primer was provided in the miScript SYBR Green PCR kit. The other primer sequences were as follows: miR-16 forward, 5'-TAGCAG CACGTAAATATTGGCG-3'; U6 reverse, 5'-ACGCTTCAC GAATTTGCGT-3' and forward, 5'-CTCGCTTCGGCAGCA CA-3'. Amplification conditions were as follows: $95^{\circ} \mathrm{C}$ for $15 \mathrm{~min}$, then 40 cycles of $95^{\circ} \mathrm{C}$ for $15 \mathrm{sec}, 58^{\circ} \mathrm{C}$ for $30 \mathrm{sec}$ and $72^{\circ} \mathrm{C}$ for $30 \mathrm{sec}$. PCR products were analyzed by agarose gel electrophoresis. The relative expression levels of miR-16 were calculated using the $2^{-\Delta \Delta C t}$ method (14).

Cell transfection. To overexpress or downregulate miR-16, miR-16 mimics (5'-UAGCAGCACFUAAAUAUUGGCG-3' and 5'-CCAAUAUUUACGUGCUGCUAUU-3') and inhibitor (5'-CGCCAAUAUUUACGUGCUGCUA-3') were synthesized by Shanghai GenePharma Co., Ltd. (Shanghai, China) and transfected into the cancer cells using Lipofectamine 2000 (Invitrogen Life Technologies). Cancer cells were harvested $24 \mathrm{~h}$ subsequent to transfection and the relative expression of miR-16 was detected by qPCR.

Cell proliferation assay. The ability of cellular proliferation was evaluated using an MTT assay. Approximately $5 \times 10^{3}$ cells were seeded into 96-well culture plates and then transfected
Table I. Clinical and pathological features of 48 patients.

\begin{tabular}{lc}
\hline Variable & Cases (n) \\
\hline Total & 48 \\
Age (years) & \\
$\geq 52$ & 29 \\
$<52$ & 19 \\
Gender & \\
Male & 30 \\
Female & 18 \\
Histological type & \\
Clear cell & 39 \\
Papillary & 9 \\
Primary tumor stage & \\
T1 & 27 \\
T2 & 19 \\
T3 and 4 & 2 \\
AJCC clinical stages & \\
I & 27 \\
II & 18 \\
III+IV & 3 \\
\hline
\end{tabular}

AJCC, American Joint Committee on Cancer.

with 5 pmol miR-16 mature molecules (mimics/inhibitor) or a negative control. At $0,24,48$ or $72 \mathrm{~h}$ following transfection, the cells were incubated with $20 \mu \mathrm{l}$ MTT solution $(5 \mu \mathrm{g} / \mathrm{ml}$; Sigma-Aldrich, St. Louis, MO, USA) for $4 \mathrm{~h}$ at $37^{\circ} \mathrm{C}$, lysed in $150 \mu 1$ dimethyl sulfoxide and agitated for $10 \mathrm{~min}$ at room temperature. The cell number was estimated by the measurement of optical density (OD) with a Model 680 microplate reader (Bio-Rad Laboratories, Hercules, CA, USA) at a dual wavelength of $490 / 630 \mathrm{~nm}$.

Flow cytometric evaluation of apoptosis. Approximately 300,000 renal cancer cells were cultured in six-well plates. At $48 \mathrm{~h}$ subsequent to transfection, cells (including floating cells) were harvested, washed twice with cold phosphate-buffered saline (Invitrogen Life Technologies) and resuspended in $100 \mu 11 \mathrm{X}$ binding buffer (Invitrogen Life Technologies), followed by the addition of $5 \mu \mathrm{l}$ annexin V-fluorescein isothiocyanate (Invitrogen Life Technologies) and $3 \mu 1$ propidium iodide (Invitrogen Life Technologies). The fluorescence of stained cells was analyzed using flow cytometry (EPICS XL; Beckman Coulter, Brea, CA, USA) within 30 min of staining, according to the manufacturer's instructions.

Wound healing assay. A wound healing assay was used to evaluate the migration ability of renal cancer cells in vitro. Approximately 350,000 cells were seeded per 12-well dish, then transfected with miR-16 mature molecules ( $80 \mathrm{pmol})$ or negative control $(80 \mathrm{pmol}) 24 \mathrm{~h}$ later. Following transfection for $5 \mathrm{~h}$, the cell monolayer was scraped using a P-20 micropipette 
A

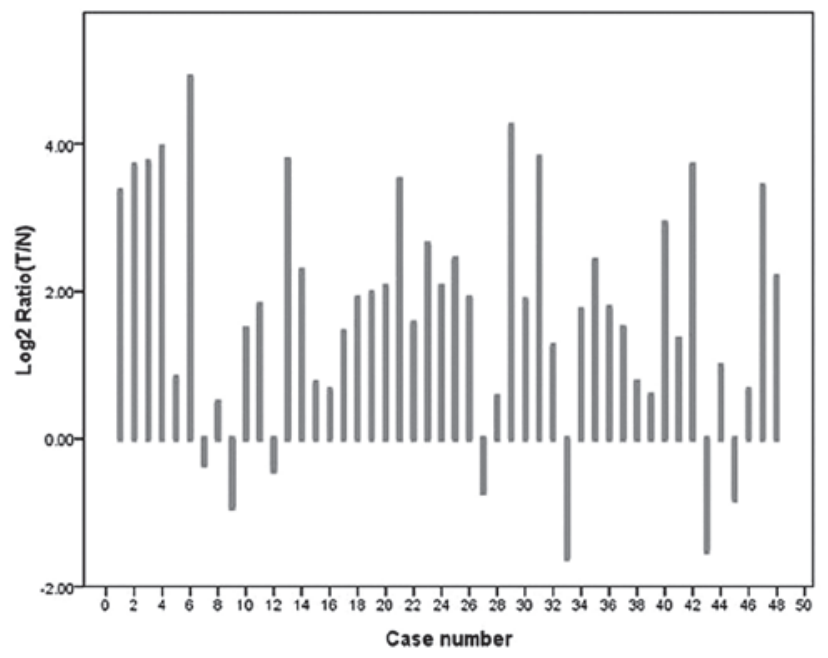

B

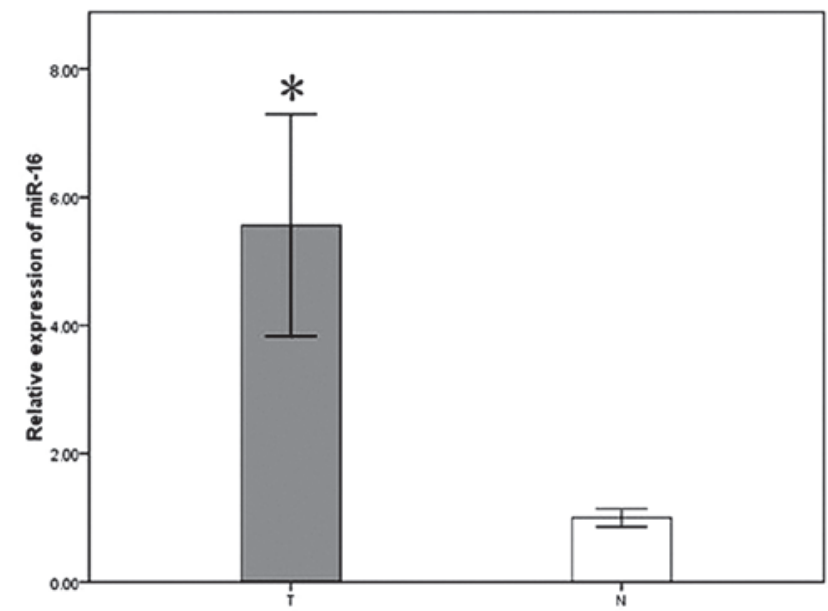

Figure 1. Expression of miR-16 in 48 paired RCC tissues and adjacent normal tissues. (A) Log2 ratios and (B) relative expression levels of miR-16 expression in 48 paired RCC and normal tissue samples. ${ }^{*} \leq 0.05$ vs. N group. $T$, RCC tumor group; $\mathrm{N}$, normal tissue group; RCC, renal cell carcinoma; miR, microR $\mathrm{NA}$.

A

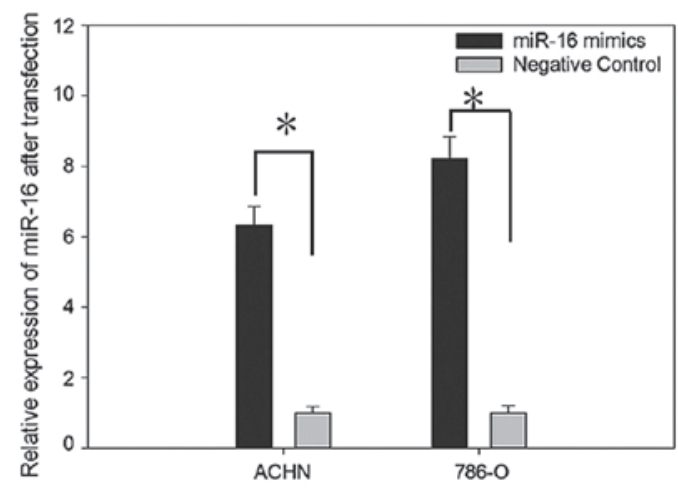

B

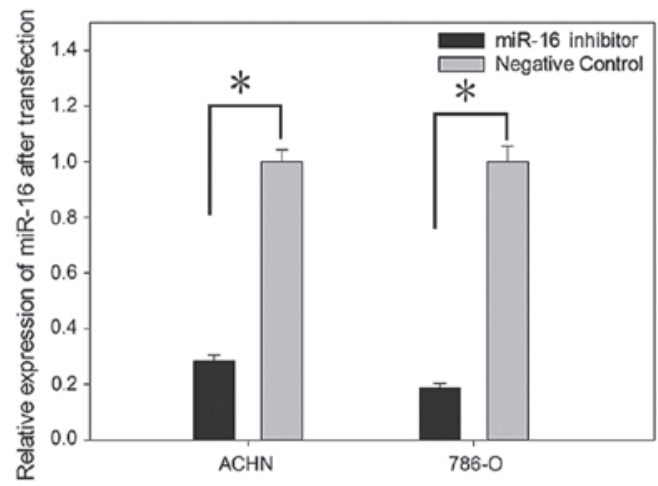

Figure 2. miR-16 expression changes after transfection were confirmed by quantitative polymerase chain reaction. Relative expression of miR-16 in ACHN and 786-O cells 24 h subsequent to transfection with (A) miR-16 mimics or (B) miR-16 inhibitor. ${ }^{*} \mathrm{P}<0.05$. miR, microRNA.

tip (Owens Corning, Toledo, OH, USA). The initial gap length $(0 \mathrm{~h})$ and the residual gap length after $24 \mathrm{~h}$ of wound-healing were calculated using the software program MIAS-2000 P3 (Leica Microsystems GmbH, Wetzlar, Germany). The experiments were performed in triplicate, repeated at least three times, and analyzed in a double-blind fashion by a minimum of two observers. The images were visualized and captured using an XSP-63XDV (Shanghai Optical Instrument Factory, Shanghai, China).

Bioinformatics analysis. The potential targets of miR-16 were predicted by combining four public algorithms, miRanda (www.microrna.org), TargetScan (www. targetscan.org), PicTar (pictar.mdc-berlin.de) and miRWalk (www.umm.uni-heidelberg.de/apps/zmf/mirwalk). Putative genes predicted by all four algorithms were accepted and candidates were selected based on the gene function.

Statistical analysis. Statistical analysis was performed using SPSS 17.0 (SPSS, Inc., Chicago, IL, USA). A paired $t$-test was used for the comparison of miR-16 expression in matched cancer and normal samples. $\mathrm{P}<0.05$ was considered to indicate a statistically significant difference.

\section{Results}

Upregulation of miR-16 in RCC tissues. Previous miR expression profiles of renal cancer have indicated that miR-16 was upregulated in these tissues $(15,16)$. In order to assess this hypothesis in the present study, qPCR was used to quantify the expression of miR-16 in 48 paired RCC tissues and adjacent normal tissues. The relative expression of miR-16 $\left[\log _{2}(\mathrm{~T} / \mathrm{N})\right]$ is exhibited in Fig. 1A. The results demonstrated that miR-16 expression levels in the RCC tissues were significantly higher than those in the paired normal tissues $(\mathrm{P}<0.05)$, as presented in Fig. 1B.

Transfection efficiency. To analyze the function of miR-16 in renal cancer, miR-16 mature molecules (mimics/inhibitor) or negative control were transfected into the ACHN and 768-O renal cancer cell lines. The relative expression of miR-16 following transfection of miR-16 mimics and inhibitor 
A

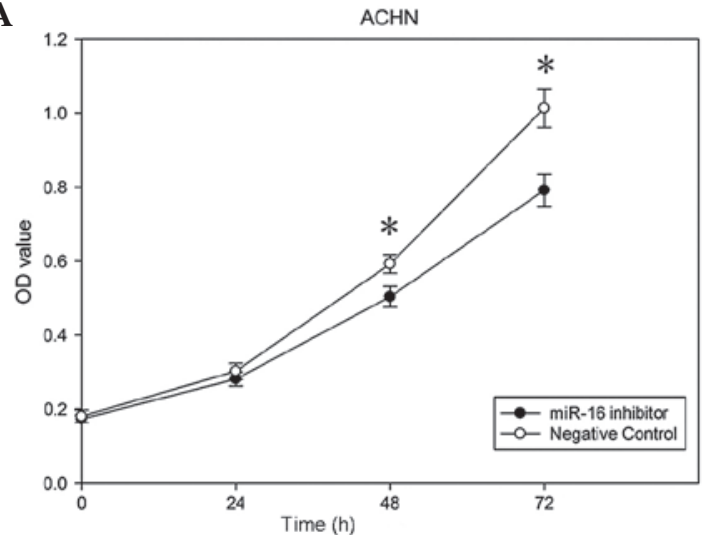

B

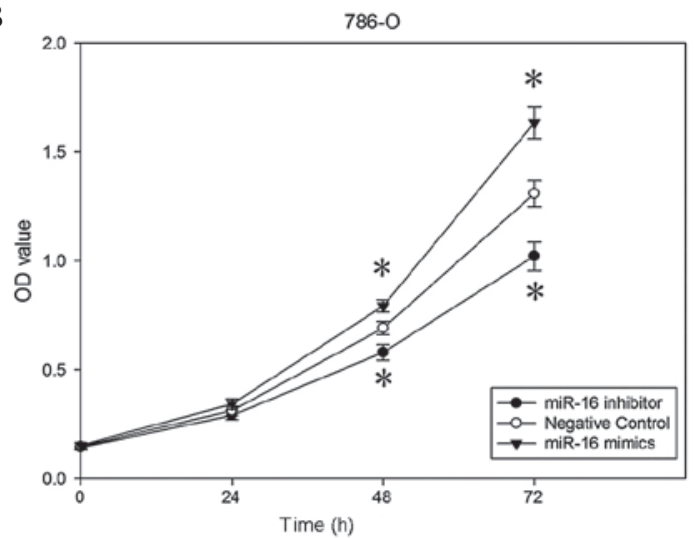

Figure 3. MTT assay indicating cell proliferation of ACHN and 786-O cells subsequent to transfection. (A) ACHN cells transfected with miR-16 inhibitor or negative control. (B) 786-O cells transfected with miR-16 mature molecules (mimics/inhibitor) or negative control. "P<0.05 vs. negative control. miR, microRNA; OD, optical density.
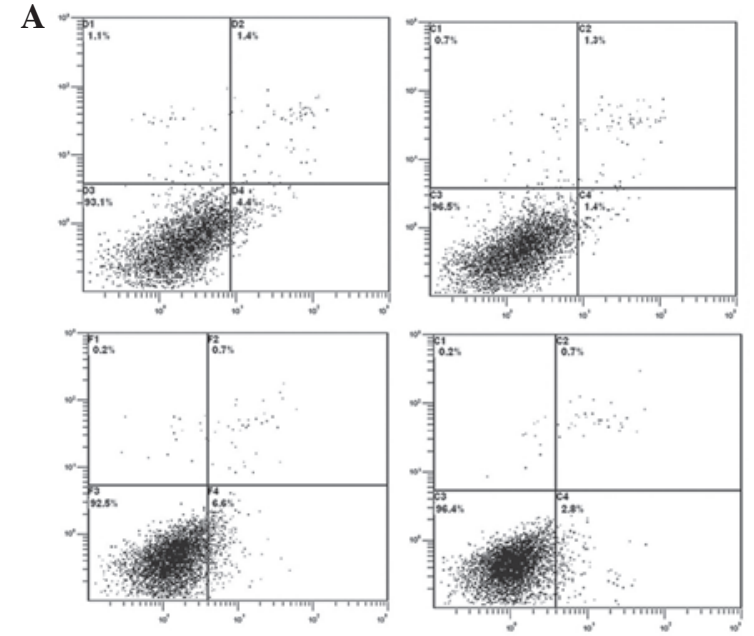

B

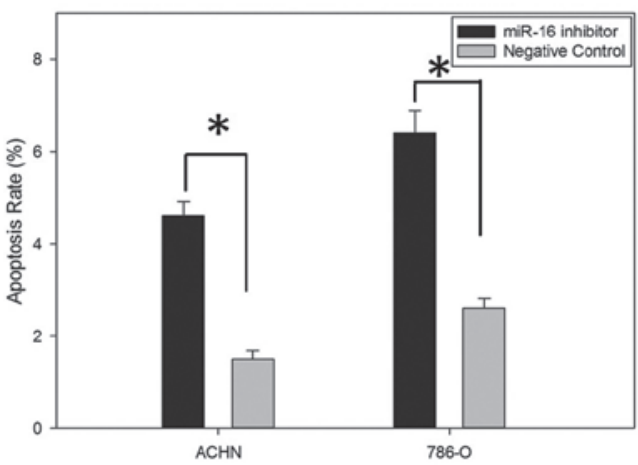

Figure 4. Flow cytometric analysis indicating levels of cell apoptosis in ACHN and 786-O cells subsequent to transfection. (A) ACHN cells transfected with miR-16 inhibitor (top left) or negative control (top right) and 786-O cells transfected with miR-16 inhibitor (bottom left) or negative control (bottom right). (B) Comparison of cell apoptosis rates in cells transfected with miR-16 inhibitor or negative control. "P<0.05 vs. negative control. miR, microRNA.

were 6.35 and 0.2638 in ACHN cells, and 8.26 and 0.1876 in 786-O cells, respectively (Fig. 2).

Effect of miR-16 on cell proliferation. Using an MTT assay, it was observed that the proliferation of 786-O cells transfected with miR-16 inhibitor was reduced by $6.06,11.84$ and $17.24 \%$ (all $\mathrm{P}<0.05$ vs. negative control), while in cells transfected with miR-16 mimics, proliferation was increased by 5.82, 15.57 and $22.42 \%$ (all $\mathrm{P}<0.05$ vs. negative control) at 24,48 and $72 \mathrm{~h}$ after transfection, respectively. In ACHN cells, proliferation was reduced by 5.2 ( $\mathrm{P}>0.05$ vs. negative control), 10.61 and $18.76 \%$ $(\mathrm{P}<0.05$ vs. negative control) following transfection with miR-16 inhibitor for 24, 48 and $72 \mathrm{~h}$, respectively. This indicated that miR-16 promotes cell proliferation in renal cancer cells (Fig. 3).

Impact of miR-16 on apoptosis. To analyze the influence of miR-16 on renal cancer cell apoptosis, a flow cytometry assay was performed to detect the rates of apoptosis of ACHN and 786-O cells following the transfection. The results indicated that apoptotic rates of ACHN cells transfected with miR-16 inhibitor were 4.4 vs. $1.4 \%$ in the negative control group, while the rates in $786-\mathrm{O}$ cells were 6.6 vs. $2.8 \%$ ( $\mathrm{P}<0.05$ vs. negative control), indicating that downregulation of miR-16 induced apoptosis in renal cancer cells (Fig. 5). Therefore, miR-16 may reduce apoptosis in renal cancer.

Influence of miR-16 on cell migration. Wound healing assays were used to assess the migration ability of renal cancer cells following transfection with miR-16 mature molecules (mimics/inhibitor) or negative control. As presented in Fig. 5, wound widths of cells transfected with miR-16 inhibitor were greater than those in the negative control group $(\mathrm{P}<0.05)$, while cells transfected with miR-16 mimics displayed increased migration, suggesting miR-16 may accelerate the migration of renal cancer cells in vitro (Fig. $5 \mathrm{C}$ ).

Target gene prediction. To explore the downstream target genes of miR-16, four algorithms were combined to predict the putative targets. BAP1 was the potential target predicted by all four algorithms simultaneously. The BAP1 3'-untranslated region (3'UTR) of the mRNA contained a complementary site for the seed sequences of miR-16 (Fig. 5D). 


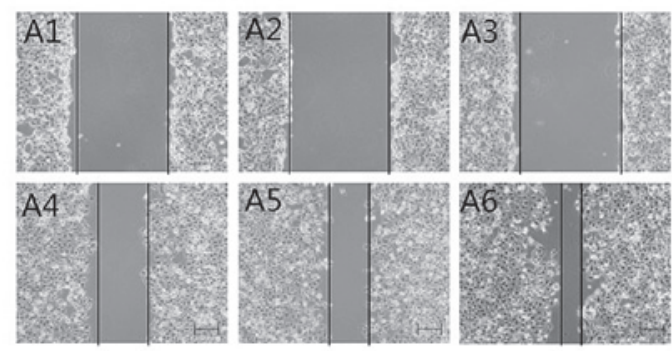

C

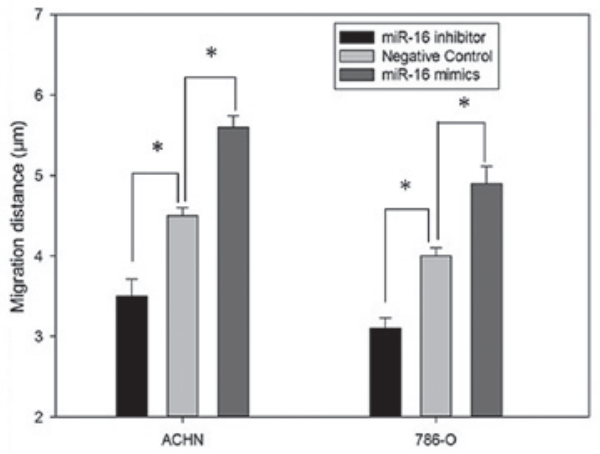

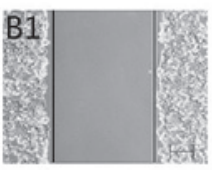

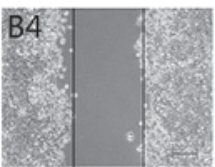

D
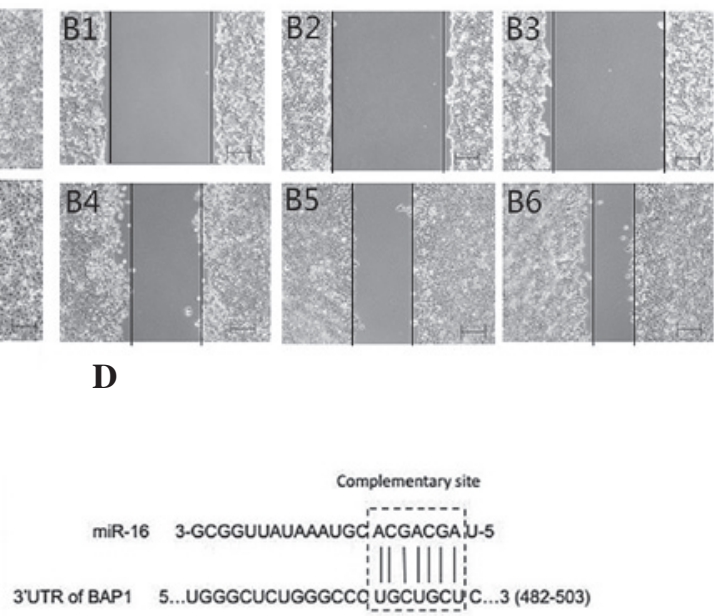

3'UTR of BAP1 5...UGGGCUCUGGGCCQ UGCUGCU:C...3 (482-503)

Figure 5. Wound healing assay of ACHN and 786-O cells $24 \mathrm{~h}$ subsequent to transfection. (A1) ACHN cells transfected with miR-16 inhibitor at $0 \mathrm{~h}$; (A2) ACHN cells transfected with negative control at $0 \mathrm{~h}$; (A3) ACHN cells transfected with miR-16 mimics at $0 \mathrm{~h}$; (A4) ACHN cells transfected with miR-16 inhibitor $24 \mathrm{~h}$ after the wounds were generated; (A5) ACHN cells transfected with negative control $24 \mathrm{~h}$ after the wounds were generated; (A6) ACHN cells transfected with miR-16 mimics $24 \mathrm{~h}$ after the wounds were generated. (B1) 786-O cells transfected with miR-16 inhibitor at 0h; (B2) 786-O cells transfected with negative control at $0 \mathrm{~h}$; (B3) 786-O cells transfected with miR-16 mimics at $0 \mathrm{~h}$; (B4) 786-O cells transfected with miR-16 inhibitor $24 \mathrm{~h}$ after the wounds were generated; (B5) 786-O cells transfected with negative control $24 \mathrm{~h}$ after the wounds were generated; (B6) 786-O cells transfected with miR-16 mimics $24 \mathrm{~h}$ after the wounds were generated. (C) Comparison of migration distance in ACHN and 786-O cells. Data are represented as the means \pm standard deviation; "P<0.05. (D) Bioinformatics prediction of the target gene of miR-16 with complementary sites. miR, microRNA; UTR, untranslated region.

\section{Discussion}

Cancer is characterized by multiple genetic changes affecting different oncogenes or tumor suppressor genes involved in cell cycle control, proliferation and apoptosis (17). The discovery of miRs and their ability to regulate multiple downstream genes indicates that they may be associated with cancer formation (18). It was estimated that $>50 \%$ of $\mathrm{miR}$ genes are located in cancer-associated genomic regions or in fragile sites, suggesting that miRs may serve an important function in the pathogenesis of human cancer (19).

Located at 13q14.3, miR-16 has been confirmed to be downregulated in chronic lymphocytic lymphoma (8). Further study revealed miR-16 expression is inversely correlated to B-cell lymphoma 2 (Bcl2) expression in this disease and that miR-16 negatively regulates $\mathrm{Bcl} 2$ at the post-transcriptional level (5). A similar mechanism has also been suggested in gastric (20) and hepatic (21) cancer. miR-16 also targets the CCND1 gene (encoding cyclin D1) and WNT3A in prostate cancer (22). These findings highlight the importance of miR-16 in carcinogenesis.

As miR-16 is involved in multiple types of neoplastic malignancy, the present study aimed to explore the potential role and effect of miR-16 in RCC. qPCR was used to quantify the expression of miR-16 in 48 samples of RCC and adjacent normal tissues. The impact of miR-16 on cell proliferation, migration and apoptosis was analyzed by transfecting miR-16 mature molecules (mimics/inhibitor) into the renal cancer cell lines 786-O and ACHN. In addition, bioinformatics analysis was performed in order to predict the target genes of miR-16.
The results of the present study demonstrated that miR-16 was upregulated in renal cancer tissue samples $(\mathrm{P}<0.05)$, which is in accordance with previous studies of miR expression profiles in RCC $(15,16)$. Downregulation of miR-16 resulted in reduced cellular proliferation, migration and increased apoptosis, while overexpression resulted in accelerated cellular proliferation and migration, suggesting that miR-16 may be characterized as an oncogene in RCC. Furthermore, bioinformatics prediction of the target gene of miR-16 indicated that BAP1 was one potential target.

In a previous study, BAP1 was observed to be mutated in $\sim 15 \%$ of clear cell RCC (ccRCC) cases and located within a $50-\mathrm{Mb}$ region on the short arm of chromosome $3 p$ that encompasses VHL and is deleted in $~ 90 \%$ of ccRCC (23). In a study by Gossage et al (24) BAP1-mutated tumors were associated with metastatic disease at presentation, advanced clinical stage and a trend towards shorter recurrence-free survival (24). Another study confirmed that the expression of BAP1 may serve as a powerful marker to predict poor outcomes in patients with cancer (25). These results indicated the critical role of BAP1 in the carcinogenesis of RCC. Upregulated miR-16 may induce cellular proliferation and migration, and reduce apoptosis by suppressing the function of BAP1.

miR-16 acts as a tumor suppressor in certain types of cancer, whereas it is an oncogene in RCC. A similar situation is observed with regards to miR-7, which has been described as a tumor suppressor in several types of human cancer, including glioblastoma, breast and lung cancer (26); however, it acts as an oncogene in RCC as previously demonstrated (27). This inconsistency may be explained by the 'imperfect complementarity' of the interactions between miRs and their target genes. 
It has been suggested that one miR can target 200 mRNAs, which may be responsible for multiple different proteins, and one mRNA can be regulated by several miRs (28).

In addition to gene regulatory functions, miRs have also been demonstrated to present significant diagnostic, prognostic and therapeutic potential. It was previously confirmed that high expression of miR-16 was associated with a significantly improved survival in advanced non-small cell lung cancer (29), and associated with ovarian cancer survival and recurrence (30). Reid et al (31) used synthetic mimics to restore miR-16 expression, which led to growth inhibition in malignant pleural mesothelioma (MPM) cell lines. Intravenous administration of miR-16 mimics in xenograft-bearing nude mice led to consistent and dose-dependent inhibition of MPM tumor growth, suggesting that restoration of expression of miR-16 may be a novel therapeutic approach for MPM.

In conclusion, the present study demonstrated for the first time, to the best of our knowledge, that miR-16 is upregulated in RCC and acts as an oncogene by accelerating cellular proliferation and migration, and by reducing levels of apoptosis. Future research of miR-16 in RCC should aim to clarify the molecular mechanism underlying RCC carcinogenesis and aid in the development of novel biomarkers and therapeutic options.

\section{Acknowledgements}

The current study was supported by the National Natural Science Foundation of China (no. 81101922), the Natural Science Foundation of Guangdong Province of China (no. S2012010008365), Medical Scientific Research Foundation of Guangdong Province of China (nos. A2012584 and A2013606), the Science and Technology Development Fund Project of Shenzhen (no. JCYJ20130402114702124) and the fund of Guangdong Key Medical Subject.

\section{References}

1. Cairns P: Renal cell carcinoma. Cancer Biomark 9: 461-473, 2010

2. Kroeze SG, Bijenhof AM, Bosch JL and Jans JJ: Diagnostic and prognostic tissuemarkers in clear cell and papillary renal cell carcinoma. Cancer Biomark 7: 261-268, 2010.

3. Pischon T, Lahmann PH, Boeing H, et al: Body size and risk of renal cell carcinoma in the European prospective investigation into cancer and nutrition (EPIC). Int J Cancer 118: 728-738, 2006

4. Eichelberg C, Junker K, Ljungberg B and Moch H: Diagnostic and prognostic molecular markers for renal cell carcinoma: a critical appraisal of the current state of research and clinical applicability. Eur Urol 55: 851-863, 2009.

5. Cimmino A, Calin GA, Fabbri M, et al: miR-15 and miR-16 induce apoptosis by targeting BCL2. Proc Natl Acad Sci USA 102: 13944-13949, 2005.

6. Wulfken LM, Moritz R, Ohlmann C, et al: MicroRNAs in renal cell carcinoma: diagnostic implications of serum miR-1233 levels. PLoS One 6: e25787, 2011.

7. Sana J, Faltejskova P, Svoboda M and Slaby O: Novel classes of non-coding RNAs and cancer. J Transl Med 10: 103, 2012.

8. Calin GA, Dumitru CD, Shimizu M, et al: Frequent deletions and down-regulation of micro- RNA genes miR15 and miR16 at 13 q14 in chronic lymphocytic leukemia. Proc Natl Acad Sci USA 99: 15524-15529, 2002 .
9. Gao X, Zhang R, Qu X, et al: MiR-15a, miR-16-1 and miR-17-92 cluster expression are linked to poor prognosis in multiple myeloma. Leuk Res 36: 1505-1509, 2012.

10. Bottoni A, Piccin D, Tagliati F, et al: miR-15a and miR-16-1 down-regulation in pituitary adenomas. J Cell Physiol 204: 280-285, 2005.

11. Teshima K, Nara M, Watanabe A, et al: Dysregulation of BMI1 and microRNA-16 collaborate to enhance an anti-apoptotic potential in the side population of refractory mantle cell lymphoma. Oncogene 33: 2191-2203, 2014.

12. Bandi N, Zbinden S, Gugger M, et al: miR-15a and miR-16 are implicated in cell cycle regulation in a Rb-dependent manner and are frequently deleted or down-regulated in non-small cell lung cancer. Cancer Res 69: 5553-5559, 2009.

13. Porkka KP,Ogg EL, Saramäki OR, et al: The miR-15a-miR-16-1 locus is homozygously deleted in a subset of prostate cancers. Genes Chromosomes Cancer 50: 499-509, 2011.

14. Schmittgen TD and Livak KJ: Analyzing real-time PCR data by the comparative $\mathrm{C}(\mathrm{T})$ method. Nat Protoc 3: 1101-1108, 2008.

15. Yi Z, Fu Y, Zhao S, Zhang X and Ma C: Differential expression of miRNA patterns in renal cell carcinoma and nontumorous tissues. J Cancer Res Clin Oncol 136: 855-862, 2010.

16. Jung M, Mollenkopf HJ, Grimm C, et al: MicroRNA profiling of clear cell renal cell cancer identifies a robust signature to define renal malignancy. J Cell Mol Med 13: 3918-3928, 2009.

17. Kosari F, Parker AS, Kube DM, et al: Clear cell renal cell carcinoma: gene expression analyses identify a potential signature for tumor aggressiveness. Clin Cancer Res 11: 5128-5139, 2005.

18. Shenouda SK and Alahari SK: MicroRNA function in cancer: oncogene or a tumor suppressor? Cancer Metastasis Rev 28: 369-378, 2009.

19. Zhang B, Pan X, Cobb GP and Anderson TA: microRNAs as oncogenes and tumor suppressors. Dev Biol 302: 1-12, 2007.

20. Xia L, Zhang D, Du R, et al: miR-15b and miR-16 modulate multidrug resistance by targeting BCL2 in human gastric cancer cells. Int J Cancer 123: 372-379, 2008

21. Guo CJ, Pan Q, Li DG, Sun H and Liu BW: miR-15b and miR-16 are implicated in activation of the rat hepatic stellate cell: An essential role for apoptosis. J Hepatol 50: 766-778, 2009.

22. Bonci D, Coppola V, Musumeci M, et al: The miR-15a-miR-16-1 cluster controls prostate cancer by targeting multiple oncogenic activities. Nat Med 14: 1271-1277, 2008.

23. Brugarolas J: PBRM1 and BAP1 as novel targets for renal cell carcinoma. Cancer J 19: 324-332, 2013.

24. Gossage L, Murtaza M, Slatter AF, et al: Clinical and pathological impact of VHL, PBRM1, BAP1, SETD2, KDM6A and JARID1c in clear cell renal cell carcinoma. Genes Chromosomes Cancer 53: 38-51, 2014.

25. Kapur P, Christie A, Raman JD, et al: BAP1 immunohistochemistry predicts outcomes in a multi-institutional cohort with clear cell renal cell carcinoma. J Urol 191: 603-610, 2014.

26. Kalinowski FC, Brown RA, Ganda C, et al: microRNA-7: A tumor suppressor miRNA with therapeutic potential. Int J Biochem Cell Biol 54: 312-317, 2014.

27. Yu Z, Ni L, Chen D, et al: Identification of miR-7 as an oncogene in renal cell carcinoma. J Mol Histol 44: 669-677, 2013.

28. Schaefer A, Jung M, Kristiansen G, et al: MicroRNAs and cancer: Current state and future perspectives in urologic oncology. Urol Oncol 28: 4-13, 2010.

29. Wang Y, Gu J, Roth JA, et al: Pathway-based serum microRNA profiling and survival in patients with advanced stage non-small cell lung cancer. Cancer Res 73: 4801X-4809, 2013.

30. Delfino KR and Rodriguez-Zas SL: Transcription factor-microRNA-target gene networks associated with ovarian cancer survival and recurrence. PLoS One 8: e58608, 2013.

31. Reid G, Pel ME, Kirschner MB, et al: Restoring expression of miR-16: a novel approach to therapy for malignant pleural mesothelioma. Ann Oncol 24: 3128-3155, 2013. 